\title{
Development of a serious computer game based training module and its integration into working at heights mine site induction - paper II
}

\author{
Phillip Stothard*, School of Mining Engineering, UNSW, Sydney, NSW 2052, Australia \\ *Corresponding author, email Phillip.stothard@curtin.edu.au
}

Anton van den Hengel, School of Computer Science, University of Adelaide, Adelaide, SA 5005, Australia

\begin{abstract}
This paper reports the findings of a collaborative project that developed and demonstrated a serious computer game (SCG) based simulation training module for mine site inductions. It is the second of two papers. The project was collaboration between the University of New South Wales, the University of Adelaide, BHP Billiton Olympic Dam Expansion, Resources and Engineering Skills Alliance, Training and Further Education South Australia and SkillsDMC. The pilot project was aimed at improving mine site inductions by developing a prototype SCG for trainers to incorporate into their regular training activities. The outcome was a high quality generic SCG that provides an interactive visualisation of an Australian mine site operation under construction. The SCG was tested under controlled conditions and subsequently deployed on site.
\end{abstract}

Keywords: Serious computer games, Instructional design, Mine education and training, Virtual Reality

\section{Introduction}

Serious Computer Games (SCG) is a relatively new term resulting from the application of computer games technology to real life situations for visualisation and education. Many simulations already developed for mining applications are based on computer games technology and are reported in Paper I [1] and the project report[2]. In earlier research efforts, a common term used for SCG was virtual reality environments (VREs). In this paper, SCG essentially means VRE and hence a technology providing three dimensional spatial environments via computer-graphics technology.

This is the second of two papers and presents and reviews the findings of the development of the instructional design within the SCG and subsequent module deployment. The project was collaboration between the University of New South Wales (UNSW) - School of Mining Engineering, the University of Adelaide - Australian Centre for Visual Technologies (ACVT), BHP Billiton Olympic Dam Expansion, Resources and Engineering Skills Alliance (RESA), TAFESA and Skills DMC. The SCG was aimed at improving mine site inductions for the hard rock mining industry in South Australia.

The 'Working at Heights' subject area was chosen as a high risk area of mine site operations and the project outcome was a high quality generic SCG that provides an interactive visualisation of an Australian mine site operation. Interaction within one of the sub-modules was comprehensively based on modern instructional design techniques. The consideration of instructional design and cognitive load theory significantly changes the interaction within the module. 
The deployment produced valuable data and the conclusions and recommendations derived from the study are summarised in the following sections. A screen shot of the Working at Heights module is shown in Figure 1 and Figure 2.

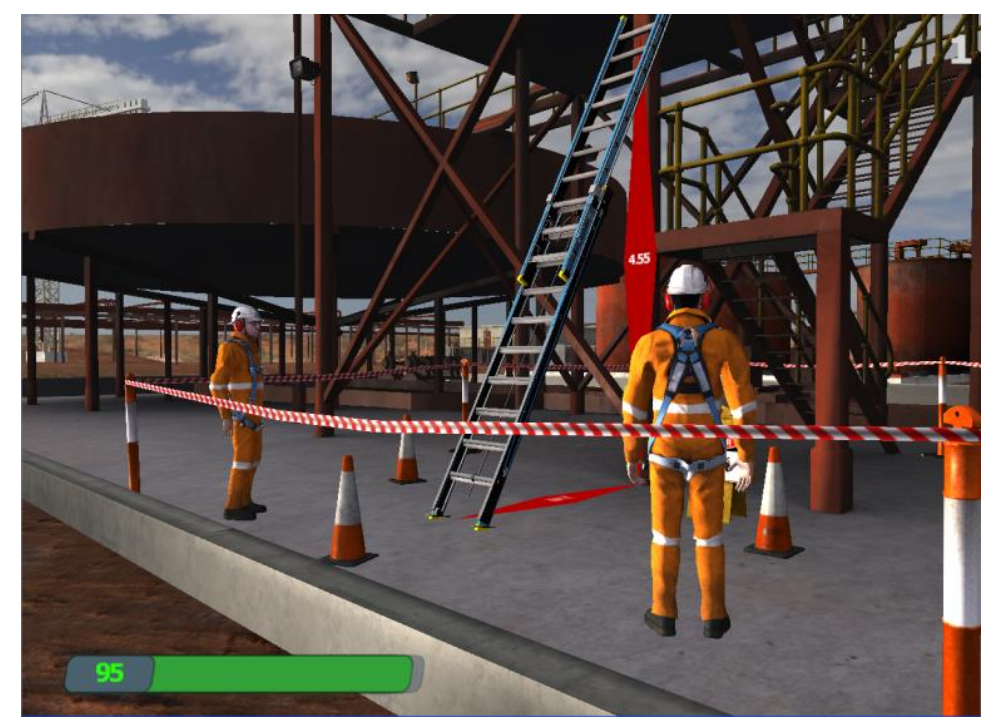

Figure 1. Screen shot of working at heights module: correcting ladder angle.

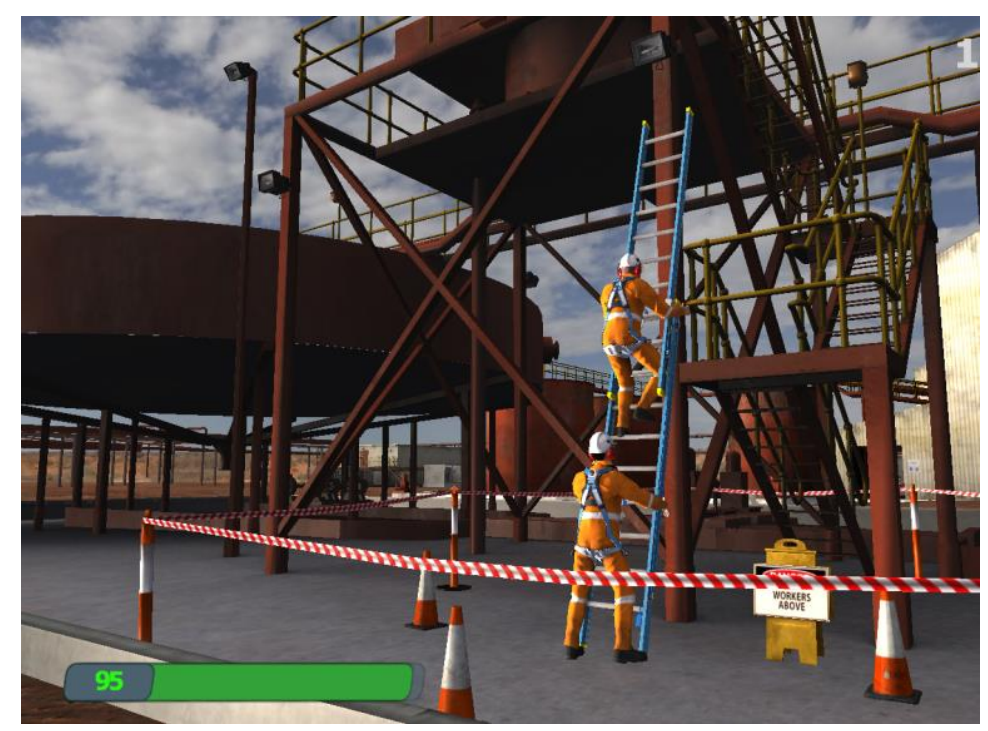

Figure 2. Screen shot of working at heights module: safe use of ladders.

\section{Serious Computer Game Evaluation}

The results of an initial prototype deployment to a group of apprentices in Adelaide are presented in paper I and the project report[1,3]. Following that deployment, a series of controlled scientific experiments that considered the SCG's instructional design was performed. A comprehensive literature survey was performed to guide the development of the instructional design within the SCG[4].

The SCG environment is such that the participant is partially or completely immersed in and able to interact with a computer generated approximation of the real world. There are a number of characteristics unique to computer generated environments that have the potential to offer superior learning experiences when 
compared to traditional classroom methods. These characteristics include immersion, presence, fidelity and learner control[5]. However, a key factor not mentioned in the literature is consistent access to realistic mine scenes for education and training. The aim of SCG is to provide the user with a site based experience that prepares the user for a real environment.

Mining is a high production environment that changes rapidly. In the case of underground mine environments and some surface mine environments, reliable and consistent access is an issue for training and there are many variables that must be considered that impact on consistency of training and education. The most prominent is visibility so that a person can actually see the environment in which he or she will work. In real life, visibility is often very poor, hence a high fidelity, high resolution model of a site can allow the trainee to experience a hazardous environment from the safety of a high quality synthetic SCG visualisation. Once this benchmark is set, all of the issues mentioned above can be evaluated and developed to deliver the necessary learning experience. However, instructional design and cognitive load theory must also be carefully considered. The course design within an SCG is extremely important for mining related applications.

\section{Controlled study at UNSW}

A controlled study was conducted at UNSW to improve the SCG and acquire some formal data on user experience and knowledge transfer and retention. A detailed analysis of the results is presented by Bennett et al[4]. The following is a summary of those experiments.

Instructional design and true learning outcomes from interacting with an SCG have often been a secondary consideration to technology. The need to integrate an SCG properly into a training or education program is essential for community acceptance and for the SCG to be utilised to its full potential. Hence, the controlled study addressed two questions.

1. Does the SCG training enable the trainee to experience an increase in human interaction (measured by levels of immersion and presence) as opposed to conventional PowerPoint training?

2. Is the SCG module a more effective training tool when compared to PowerPoint training methods showing similar high quality images?

Kirkpatrick's[6] learning evaluation framework was used and three of the four evaluation levels were adopted. In this model, each successive evaluation level builds on information from a lower level. Level one measures how participants react to a training program and attempts to answer questions regarding the participants' perceptions (i.e. did they like it? was the material relevant to their work?). Level two attempts to assess the amount of learning that has occurred. Specifically, it assesses the extent that students have advanced in skills, knowledge or attitudes. Retention tests are often used at this level. Level three measures the change in the learners' behaviour due to the training program. This level examines whether the newly acquired skills, knowledge or attitudes are being used by the learner. Level four considers the results and what are the organisational benefits resulting from training?

Sixty novices to mining were studied. Each was randomly allocated to one instructional condition, PowerPoint in a group setting (PP), SCG in a group setting (SG) or SCG in an individual setting (SI). Mean age was 19.72 years, and $55 \%$ were female. Comparisons were made across the instructional conditions relating to measures of knowledge, immersive tendency, presence and satisfaction. The display system was a large flat screen (2.56 by $1.6 \mathrm{~m}$ ) with stereo speakers and a joystick for interaction. To reduce extraneous processing and thus cognitive load, three evidence based instructional principles were adopted:6 the redundancy principal, which states that people learn best from animation and narration as opposed to animation, narration and on-screen text; signalling, whereby learning is enhanced when the learners' attention is guided towards the relevant 
material by highlighting the essential material in the lesson; and the temporal contiguity principal, which states that learning improves when corresponding narration and animation are displayed simultaneously, as opposed to successively. As a result, in the simulation module, on-screen written text was used sparingly to signal relevant material and was only displayed after the simultaneous computer based narration and animation had been presented. To foster essential processing, the segmenting principal[7] was also followed. This principle states that when narrated animation is presented in learner paced segments rather than as a continuous presentation, learning is improved. Hence, at the end of each module segment, a CONTINUE button appeared.

Schema formation was also considered during the design phase of the SCG instruction[7]. Learning is most efficient, effective and satisfying when the participant is able to arrange information from the instructional programs themselves instead of learning through rote methods. A mix of instruction and practice was developed resulted and was achieved by first providing safety information as instruction and second, to promote practice, allowing the participants to test their knowledge by asking them to answer a range of multiple choice questions.

The PowerPoint based system consisted of Microsoft PowerPoint software in addition to the same projector, large flat screen and PC used in the SCG instruction. Instead of using the joystick, the computer mouse was used for interaction. Static screen shots from the SCG module were presented and narration was given by the experimenter. Printed text was again used to signal relevant material, but, in this case, was presented simultaneously with the images and narration. The safety content provided was identical to the SCG instruction. Furthermore, the structure of the PowerPoint created for the instruction was similar to the SCG instruction, with the first half of the instruction providing information on safety and the latter half testing the participants' knowledge via multiple choice questions.

An immersive tendency questionnaire (ITQ) measured participant involvement with the visual material [8]. A presence questionnaire (PQ) measured participant perception of the instructional system. A satisfaction survey asked participants to rate their level of interest, control and perceived difficulty of the material in the instructional environment. The satisfaction survey also served to evaluate level 1 of Kirkpatrick's learning evaluation model. Participants were given computerised knowledge tests which served as a measure of learning. The first test included 17 multiple choice questions. The second test was administered to participants 1 week after the training via email using Microsoft Word.

Participants were randomly assigned to an instructional condition (PP, SG and SI). The 'group' conditions comprised 3-5 people seated together. The experimenter controlled the session via the mouse or joystick. During the 'individual' condition, participants trained independently and controlled the joystick. Training was given and followed by a 10 min break. A small PC screen 'recall and transfer' test was then administered and, finally, the satisfaction survey was completed. Exactly 1 week later, the participants were required to complete the final retention test via email. A summary of the instructional conditions used in the experiment is displayed in Table 1.

\section{Results: evaluating SCG}

Comparisons were made across the instructional groups on each of the dependent measures: presence, knowledge and satisfaction. For each measure, a multivariate analysis of variance was conducted. Two comparisons were conducted, the first examining the difference between the PowerPoint and simulation in a group setting (PP versus SG) conditions and the second examining the difference between simulation in a group versus simulation in an individual setting (SG versus SI). 


\section{PowerPoint versus SCG in group setting}

The ITQ data are presented in Table 2. No significant differences were found between the two groups indicating that the overall tendency of the participants to be involved in visual material was similar across both groups. The PQ data are presented in Table 3. Significant differences were found between the two instructional groups. Participants exposed to the SCG experienced a significantly larger degree of: control in the training environment; sensory information transmitted from the environment; and realism and authenticity from the training scenes than did the PP group. The SG group level of presence was significantly greater than that experienced by the PP group. Distraction was not significantly different across the two groups, suggesting that participants' willingness to concentrate on the training and ignore distractions was similar across both instructional groups. Knowledge and retention test scores are displayed in Table 4. No significant difference was found between the PP and SG groups for the knowledge test. The difference in retention between the PowerPoint and SG groups was also very small. Across the satisfaction survey, only one significant difference was found. The question, 'Please indicate the perceived level of realism in the visual material', suggests that the individuals in the SG group perceived the realism of the training to be greater than the PP group, a feature that is a goal of SCG training and education.

\begin{tabular}{lcc} 
Table 2 & $\begin{array}{l}\text { Means } \\
\text { deviations (MD) }\end{array}$ & $\begin{array}{c}\text { and } \text { on ITQ for PP and SG conditions } \\
\text { corresponding }\end{array}$ \\
\hline & ITQ & \\
Group & Mean & SD \\
\hline PP & 110.80 & 16.05 \\
SG & 114.90 & 13.97 \\
\hline
\end{tabular}

Table 3 Means (M) and standard deviations (SD) on PQ for PP and SG conditions

\begin{tabular}{|c|c|c|c|c|c|c|c|c|c|c|}
\hline \multirow[b]{3}{*}{ Group } & \multicolumn{10}{|l|}{$\mathrm{PQ}$} \\
\hline & \multicolumn{2}{|c|}{ Control (70) } & \multicolumn{2}{|c|}{ Sensory (77) } & \multicolumn{2}{|c|}{ Distraction (35) } & \multicolumn{2}{|c|}{ Realism (49) } & \multicolumn{2}{|c|}{ Total (224) } \\
\hline & M & SD & $M$ & SD & $M$ & SD & M & SD & M & SD \\
\hline $\mathrm{PP}$ & 39.50 & $9 \cdot 49$ & $31 \cdot 15$ & $10 \cdot 12$ & $25 \cdot 15$ & $4 \cdot 61$ & 24.05 & 6.05 & $121 \cdot 15$ & 18.97 \\
\hline SG & $46 \cdot 90$ & $10 \cdot 33$ & 44.95 & $10 \cdot 70$ & 23.90 & 5.59 & 28.50 & 4.98 & $142 \cdot 45$ & 24.93 \\
\hline
\end{tabular}


Table 4 Means (M), standard deviations (SD) and percentage correct values ( $\%$ C) on knowledge and retention for PP and SG conditions

\begin{tabular}{llllllll}
\hline & \multicolumn{3}{l}{ Knowledge } & \multicolumn{5}{l}{ Retention } \\
\cline { 2 - 4 } \cline { 6 - 8 } Group & M & SD & \% C & & M & SD & $\%$ C \\
\hline PP & 14.60 & 1.96 & $85.9 \%$ & & 14.15 & 1.81 & $83.2 \%$ \\
SG & 14.45 & 1.64 & $85.0 \%$ & & 14.05 & 1.47 & $82.6 \%$ \\
\hline
\end{tabular}

Table 5 Means (M) and standard deviations (SD) on ITQ for SG and SI groups

\begin{tabular}{lll}
\hline & \multicolumn{2}{l}{ ITQ } \\
\cline { 2 - 3 } Group & $\mathrm{M}$ & $\mathrm{SD}$ \\
\hline $\mathrm{SG}$ & 114.90 & 13.97 \\
$\mathrm{SI}$ & $120 \cdot 00$ & 14.19 \\
\hline
\end{tabular}

\section{Group SCG versus individual SCG}

The ITQ data for both SCG groups are presented in Table 5. Once again no significant differences for immersive tendency were found. The PQ data for both SCG groups are displayed in Table 6. No meaningful variations between the two SCG groups were found. Furthermore, no significant difference appeared across the two groups for the overall PQ data. Table 7 presents the data for the knowledge tests. The individual SCG shows a significantly higher score for both the knowledge and retention tests when compared to the SG group. Retention loss was present in both groups, but not significantly different between them. In the SI group, higher scores are encouraging and suggest some increased engagement with the SCG.

To examine whether a relationship existed between the $P Q$ and the two knowledge tests, bivariate correlation analyses were conducted. Table 8 shows that overall PQ failed to correlate significantly with the knowledge tests. Significant correlation did occur between the distraction subscale and the two knowledge tests in the simulation group condition. This suggests that the more the observer can ignore distractions, the higher the knowledge and retention. While the sample is small for such an analysis $(n=20)$, this result suggests that this group was able to concentrate on the SCG environment, and as a result, this led to an improvement in their knowledge.

Table 6 Means (M) and standard deviations (SD) on PQ for SG and SI groups

\begin{tabular}{|c|c|c|c|c|c|c|c|c|c|c|}
\hline \multirow{2}{*}{ Group } & \multicolumn{10}{|l|}{$P Q$} \\
\hline & \multicolumn{2}{|c|}{ Control (70) } & \multicolumn{2}{|c|}{ Sensory (77) } & \multicolumn{2}{|c|}{ Distraction (35) } & \multicolumn{2}{|c|}{ Realism (49) } & \multicolumn{2}{|c|}{ Total (224) } \\
\hline SG & $46 \cdot 90$ & $10 \cdot 33$ & 44.95 & $10 \cdot 70$ & 23.90 & 5.59 & 28.50 & 4.98 & $142 \cdot 45$ & 24.93 \\
\hline SI & $47 \cdot 80$ & $6 \cdot 80$ & $47 \cdot 70$ & $9 \cdot 31$ & $24 \cdot 15$ & $2 \cdot 25$ & 28.9 & $5 \cdot 82$ & $147 \cdot 10$ & 17.91 \\
\hline
\end{tabular}




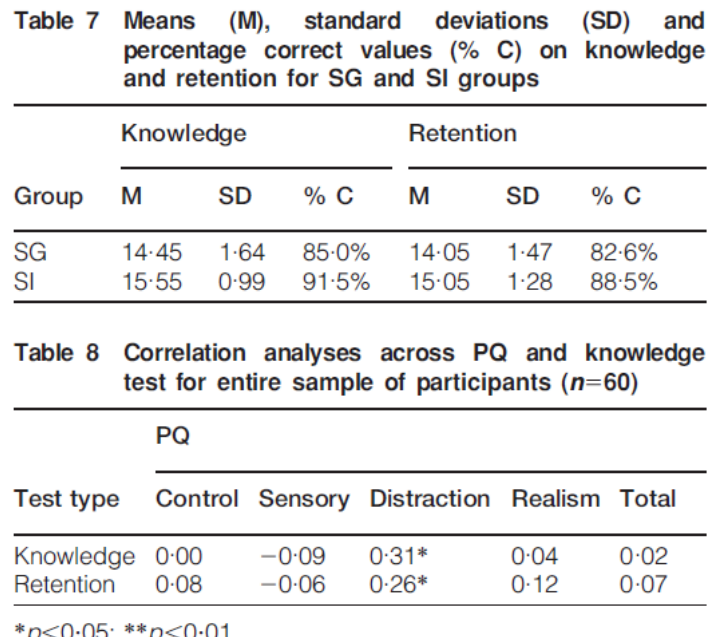

\section{Deployment at Olympic Dam site}

Olympic Dam kindly accommodated a site deployment and due to time restrictions, uncontrollable variables and logistical issues, only a general satisfaction survey was conducted. The deployment took place in a classroom environment at the 'pilot plant' training site. The course was trainer led as shown in Fig. 3.

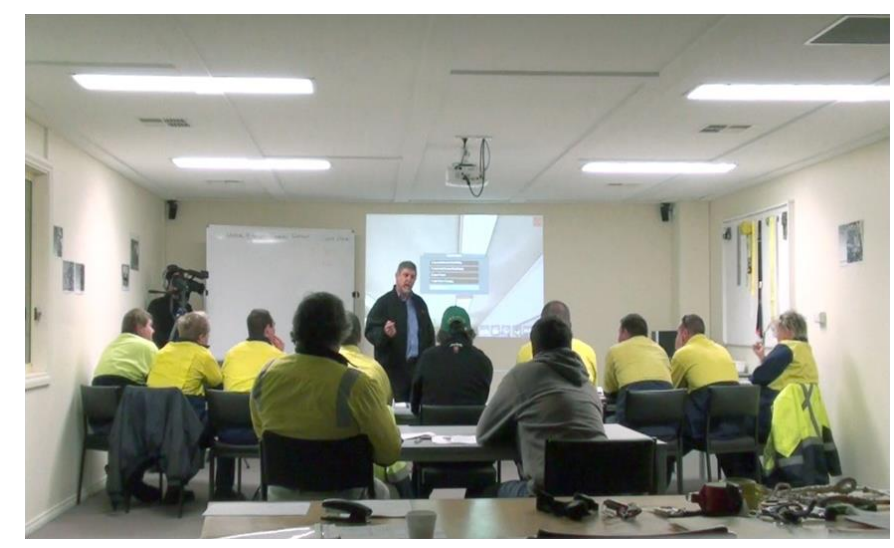

Figure 3. Intergration of module into mine site inductions.

A contract trainer exposed mine workers to the simulation over a period of 4 weeks and due to limited time, was asked to collect satisfaction/rating data only. The aim was to use the same lesson plan as used in Adelaide and simply establish how the trainees felt about the simulation. This provided an evaluation of the SCG at level 1 of Kirkpatrick's[6] model. The SCG referred to in the survey is the 'change a light globe using ladders'. This was one of the five sub-modules improved by UNSW following the Adelaide experimental deployment. Screen shots are shown in Figs. 1 and 2.

The results from the Olympic Dam satisfaction survey are shown in Figs. 4 and 5. The interviewed miners prefer practical based training and also like the combination of lecture and computer based training, which includes the SCG. Very few like pure computer or video based training and none likes pure lecture based training. This is not unexpected as these participants are practical, site based people. 


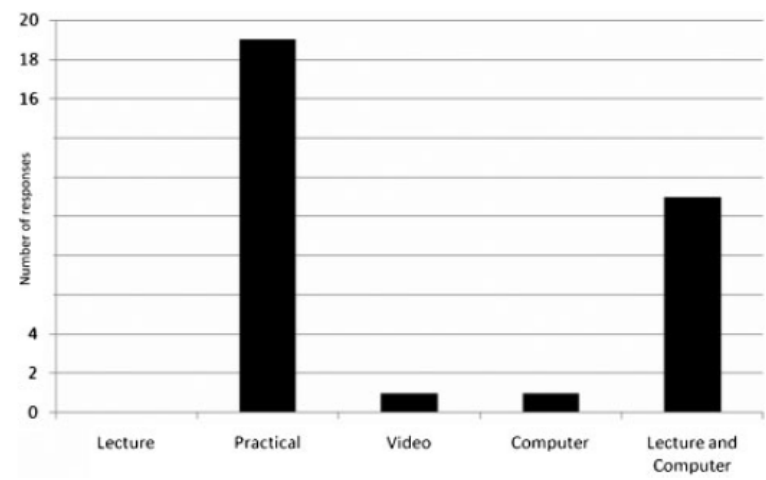

Figure 4. Olympic Dam miners: preferred method of teaching.

Figure 5 shows the satisfaction data for the Olympic Dam miners. Most found that the training was engaging and interactive and also found that they learned something from the training, although there were people who did report that they did not learn anything at all. The Olympic Dam miners were confident that they could safely change a light globe at height on an open cut mine, using ladders, after the training. They did not find the material difficult to learn and the skew is towards little effort being involved. The perceived level of realism was high and the data would suggest that the participants generally liked the SCG.

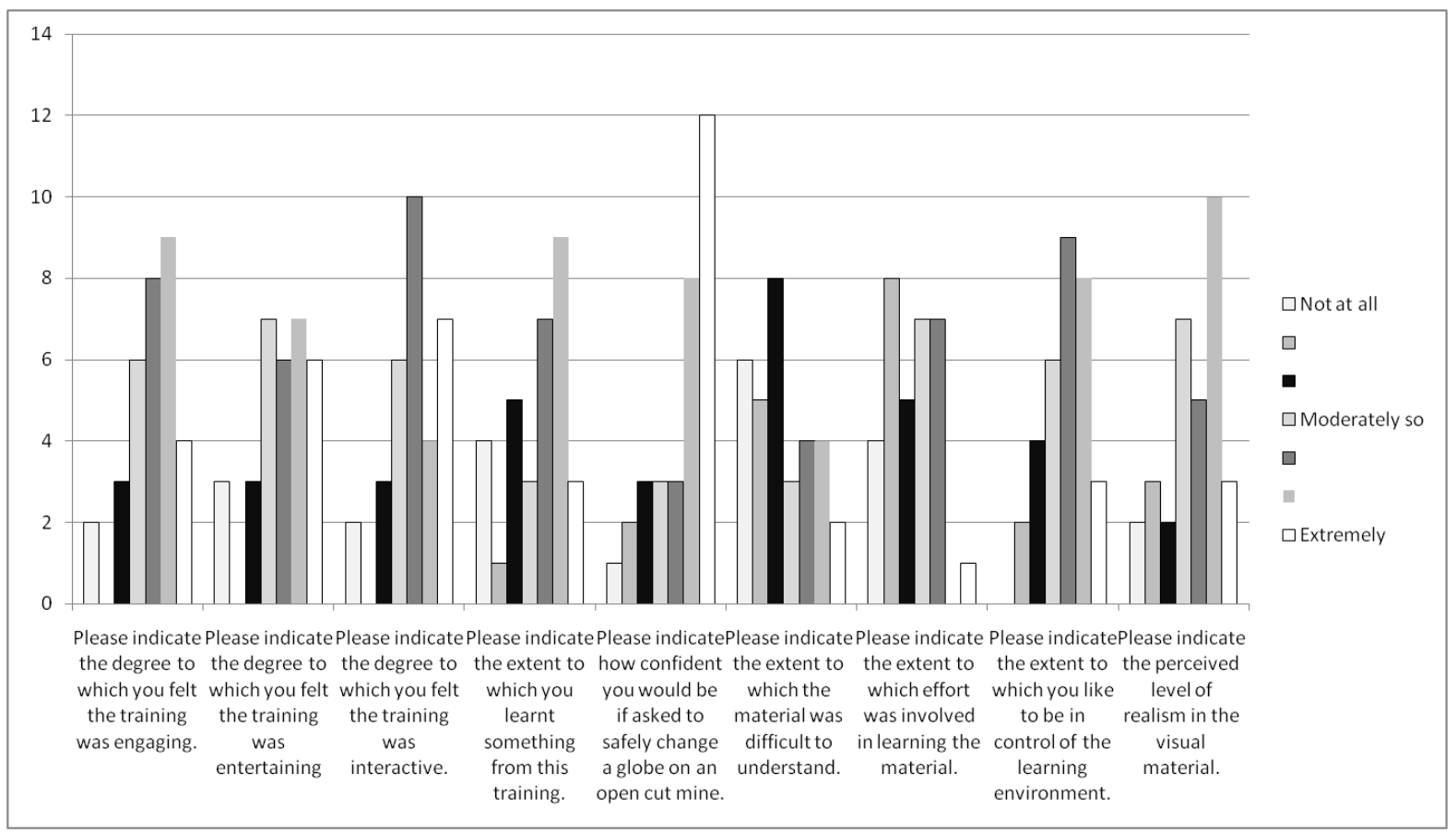

Figure 5. Olympic Dam working at heights course: satisfaction/rating data

\section{Conclusions and recommendations}

Following an initial deployment as described in paper I, the SCG module was modified and evaluated under controlled conditions and then deployed onto the Olympic Dam Mine Site as a pilot study. The controlled experiments asked two key questions: 
1. Does the SCG training enable the trainee to experience an increase in human interaction (measured by levels of immersion and presence) as opposed to conventional PowerPoint training?

2. Is the SCG module a more effective training tool when compared to PowerPoint training methods showing similar high quality images?

The experiments found that the SCG training module facilitated an increase in presence when compared to the PowerPoint instruction under controlled conditions but increased presence did not promote an increase in learning. However, it is a site based experience that the SCG is ultimately trying to achieve and the increased level of presence from the SCG would suggest that this is beginning to be achieved, but still requires development. The SCG has provided an increase in human interaction compared to PowerPoint in the SCG SI condition.

Under controlled conditions, the SCG was only marginally better as a training tool under group conditions when similar images in the SI, SG and PP conditions were used. However, when the trainees were allowed to interact with the SCG themselves under the SI condition, there was an increase above the PP condition and the SG condition. This is encouraging and prompts the need to develop interactive SCG under SI conditions, which may lead to improved training tools. Unfortunately, under the limited deployment at Olympic Dam and the need for a more formal integration, the SCG can only be said to be marginally better than the PP conditions. This requires more investigation. The sub-modules that make up the working at heights module were deployed on site as a group training course. Trainees working at Olympic Dam experienced all the SCG training modules and the data collected show that the group preferred practical training and then combined lecture and computer based training.

The trainees were confident that they could perform the light globe operation following the training in that module and generally thought they had learned something about safety. The material was easy for the Olympic Dam population to learn and this may be due to their previous experience or that the level of the simulation was too low. Level of difficulty within modules requires further work.

The trainees want control of their training. This suggests that the application of SCG to mine worker training should be done in a fashion where relevant practical aspects of their daily work and training requirements should be replicated so that they utilise and apply that practical experience within the simulation. That is, the SCG should get them to solve problem within the environment and force trainees to interact with the environment in much more detail as opposed to being an observer within a trainer led exercise.

In summary, the pilot module was deployed to site successfully and is available for training if required by the Olympic Dam site; however, the application of SCG simulation on mine sites and their integration into training courses requires much more work. The consideration of the instructional design and its integration into mine site training operations also requires further research and a formal on-site implementation of simulation technology in purposely built facilities should also be considered in any future deployment.

\section{References}

1. Stothard, P.M. and A. van den Hengel, Development of serious computer game based training module and its integration into working at heights mine site induction-Part $I$. AusIMM International Transactions,, 2010. Volume 119, (Number 2,): p. pp. 68-78(11).

2. Stothard, P. and A. van-den-Hengel, Feasibility Study: Development and demonstration of virtual reality aimulation training for the BHPB Olympic Dam Site Inductions, 2009, UNSW Sydney. 
3. Stothard, P. and A. van-den-Hengel, Feasibility Study: Development and demonstration of virtual reality simulation training for the BHPB Olympic Dam Site Inductions, 2009, UNSW Sydney.

4. Bennett, L., P. Stothard, and J. Kehoe. Evaluating the Effectiveness of Virtual Reality Learning in a Mining Context. in SimTect 2010. 2010. Brisbane Australia: Simulation Industry Association of Australia.

5. Dalgarno, B., J. Hedberg, and B. Harper. The contribution of 3D environments to conceptual understanding. in Proceedings of the 19th Annual Conference of the Australasian Society for Computers in Learning in Tertiary Education 2002. Auckland, NZ: UNITEC Institute of Technology.

6. Kirkpatrick, D.L., Techniques for evaluating training programs. Journal of the American Society of Training Directors, 1959. 13(9): p. 21-26.

7. Mayer, R.E., et al., Increased interestingness of extraneous details in a multimedia science presentation leads to decreased learning. . Journal of Experimental Psychology: Applied, 2008. 14(4): p. 329-339.

8. Witmer, B.G. and M.J. \& Singer, Measuring presence in virtual environments: A presence questionnaire. . Presence: Teleoperators and Virtual Environments, 1995. 7: p. 225-240. 